\title{
La rhétorique antiphilosophique face au « cas Fénelon ": Cruzamante ou la Sainte Amante de la Croix de Marie-Françoise Loquet (1786)
}

\section{Agathe Mezzadri-Guedj}

\section{(2) OpenEdition}

Journals

Electronic version

URL: http://journals.openedition.org/rhetorique/846

DOI: $10.4000 /$ rhetorique.846

ISSN: 2270-6909

Publisher

UGA Éditions/Université Grenoble Alpes

Printed version

ISBN: 978-2-37747-066-2

Electronic reference

Agathe Mezzadri-Guedj, « La rhétorique antiphilosophique face au «cas Fénelon » : Cruzamante ou la Sainte Amante de la Croix de Marie-Françoise Loquet (1786) », Exercices de rhétorique [Online], 12 | 2019, Online since 03 February 2019, connection on 12 September 2020. URL : http://

journals.openedition.org/rhetorique/846 ; DOI : https://doi.org/10.4000/rhetorique.846

This text was automatically generated on 12 September 2020 .

\section{c) () (2) (2)}

Les contenus de la revue Exercices de rhétorique sont mis à disposition selon les termes de la Licence Creative Commons Attribution - Pas d'Utilisation Commerciale - Partage dans les Mêmes Conditions 4.0 International. 


\title{
La rhétorique antiphilosophique face au « cas Fénelon »: Cruzamante ou la Sainte Amante de la Croix de Marie-Françoise Loquet (1786)
}

\author{
Agathe Mezzadri-Guedj
}

\begin{abstract}
Le prisonnier doit aimanter vers sa personne toutes les tensions intérieures, toutes les haines et rancunes accumulées. On lui demande de transformer par sa mort toute cette violence maléfique en un sacré bénéfique, de rendre sa vigueur à un ordre culturel déprimé et fatigué, [...] de reproduire ce qui s'est passé la première fois, de renouveler une fois de plus l'unanimité qui s'est faite et refaite autour de la victime émissaire ${ }^{1}$.
\end{abstract}

2 En France, les années 1770-1790 voient la publication de nombreux romans destinés à sauvegarder la foi catholique. Ces apologues prennent part à ce que Darrin M. Mcmahon appelle un "Catholic Counter-Enlightment ${ }^{2}$ ». De fait, le mouvement complexe et protéiforme des "Anti-Lumières " «émane souvent des représentants de l'Église catholique ${ }^{3}$ ", et s'oppose globalement aux " incrédules ${ }^{4} »$ : "Incredulity, in short, was a force ravaging society from top to bottom ${ }^{5}$ " ( Bref, l'incrédulité était une force ravageant la société de haut en bas »). Didier Masseau rappelle que l'Assemblée du Clergé tente même, en 1775, de constituer un front uni face aux philosophes : " une Société des gens de lettres pour la défense de la foi », " mais le projet avorte, l'activité des apologistes n'étant pas suffisamment concertée et peut-être trop tardive ${ }^{6} »$.

Quelques années auparavant, Fréron, critique littéraire, pose les principes d'un « roman antiphilosophique »: avant tout didactique, le roman est chargé de prévenir 
ses lecteurs du danger philosophique, tout en veillant à ne pas donner de ce danger une représentation trop attrayante ${ }^{7}$.

4 Cette approche n'est pas neuve. Le paradoxe de " condamne[r] le roman par principe ", tout en lui accordant une "utilité morale » remonte aux Aventures de Télémaque de Fénelon ${ }^{8}$.

5 Toutefois, en cette deuxième moitié $\mathrm{du} \mathrm{xVIII}^{\mathrm{e}}$ siècle, l'enjeu didactique revêt une urgence plus nette qu'en 1699. Les romanciers apologistes sont pressés par une rhétorique philosophique antisystème avant la lettre, qui s'attaque aux piliers traditionnels et institutionnels tenant l'édifice de leurs représentations morales, idéologiques et esthétiques. Dans leurs écrits, la menace politique se traduit en primat donné à la rhétorique chrétienne sur la poétique romanesque, quitte à produire des œuvres de circonstance. Nicolas Brucker le dit sans ambages : « la moralité est le critère dominant » de ce "roman antiphilosophique" " et "Avec des nuances selon les cas, il faut admettre que ces textes ont mal survécu au contexte polémique qui les a vus naître ${ }^{10} »$.

6 Si l'on porte nos regards sur les romans de femmes à visée antiphilosophique - Madame Leprince de Beaumont, Madame de Genlis, et Marie-Françoise Loquet, par exemple -, l'ensemble frappe par l'hétérogénéité formelle et thématique, d'une part, et par un même refus implicite du passage d'un régime rhétorique à un régime poétique de la fiction narrative, d'autre part ; à moins de risquer un rejet ecclésiastique ${ }^{11}$. Les romans antiphilosophiques féminins sont des régimes fictionnels a minima. Les Américaines de Marie Leprince de Beaumont, par exemple, creusent une épistémè rhétorique triomphante que la conteuse qualifiera ironiquement plus tard de «style maudit ${ }^{12}$ ». Les personnages échangent des arguments logiques (souvent empruntés à la Démonstration de l'existence de Dieu de Fénelon ${ }^{13}$ et dirigés contre le pyrrhonisme ${ }^{14}$ ), des techniques d'argumentation ${ }^{15}$, et de courtes narrations enchâssées dont l'utilité didactique est si évidente que la valeur poétique en est évacuée ${ }^{16}$.

7 Dans cette production, un apologue publié trois ans avant la Révolution française se distingue. Cruzamante de Marie-Françoise Loquet n'est pas seulement l'un des «quatre romans dévots, égrainés entre 1781 et 1788 , qui illustrent les atermoiements du monde catholique face au recours à la fiction ${ }^{17} »$. Roman d'apprentissage mystique nourri de modèles littéraires, Cruzamante nous renseigne sur un enjeu idéologique et stylistique majeur des romanciers antiphilosophes : se situer par rapport au « cas Fénelon ».

Dans Cruzamante, l'orpheline de mère, puis de père, Jeanne de la Croix remotive sémantiquement le nom du mystique espagnol Jean de la Croix en devenant, à la suite de Jésus, « une véritable crucifiée ${ }^{18}$ » et « fidèle imitatrice [du] Sauveur sur la croix ${ }^{19}$ ». L'incipit explicite à la fois le sens de l'onomastique et l'argument moral unique des pages qui suivent : « Cette héroïne de la croix, naquit à la Vera-Cruz, un Vendredi saint, on ne sait de quelle année. Tout annonçait la glorieuse destinée de cet enfant. Son état d'infirmité, le nom de sa ville, le jour de sa naissance, les épreuves que le Ciel envoya à sa famille, furent autant de présages, qu'une nouvelle Amante d'un Homme-Dieu crucifié paraissait sur la terre ${ }^{20} »$.

9 Si ce parcours victimaire illustre parfaitement les analyses intemporelles de René Girard sur «la violence et le sacré » où "l'ordre culturel » s'origine invariablement dans «[1]es métaphores de rupture », précisément la "mort» d'un "personnage exceptionnel ${ }^{21} »$ (Cruzamante est toujours « affamée de souffrances $\left.{ }^{22} »\right)$, les supplices et tentations auxquelles la jeune femme doit faire face sont de l'époque de Marie- 
Françoise Loquet. La quête puis l'ascension du "Monte-Doloroso", fournissent à Cruzamante l'occasion de repousser différentes allégories des ennemis extérieurs de la religion : le « chaos de [...] contradictions ${ }^{23}$ » des philosophes incrédules s'incarne dans «l'Esprit du Monde », l'« Amour des richesses », le « Murmure », « l'Impatience », la "Gourmandise ", la «Superbe», et la «Calomnie ». Cruzamante doit aussi se confronter aux ennemis intérieurs de la religion : la "Pusillanimité », la « Tiédeur », le « Respect humain », la «Prudence humaine », ou encore, la «Paresse», l'« Amour du repos" et le "Dégoût dans le service de Dieu». Ces derniers sont les plus insidieux, d'autant plus quand ils évoquent à la femme apologiste à la fois un modèle - le succès éditorial du Télémaque - et un contre-modèle- la condamnation papale des Maximes des saints.

10 C'est cet aspect " pour et contre Fénelon » qui saute aux yeux du lecteur de Cruzamante, ou la Sainte Amante de la croix. En effet, en cette période où le jeune public féminin menace de se tourner vers la concurrence philosophique, Marie-Françoise Loquet propose ce que le marketing nomme un rebranding - c'est-à-dire un repositionnement du roman d'apprentissage chrétien, en se différenciant du modèle français popularisé par Fénelon. Pour Lorenzo Rustighi, Marie-Françoise Loquet appartient «à sa manière à une tradition 'quasi quiétiste ${ }^{\prime 2}$ ». C'est cette "manière » et ce "quasi » que l'on souhaite ici préciser, tant la visée premièrement rhétorique, voire communicationnelle de Cruzamante s'effectue en dialogue permanent avec les codes féneloniens. En termes marketing, pour différencier la foi catholique de la menace philosophique, MarieFrançoise Loquet se positionne par rapport au leader sur le marché de l'apologue: Fénelon.

\section{Le « cas Fénelon » pour les femmes des anti- Lumières}

11 Le véritable Mentor ${ }^{25}$, Mentor vertueux ${ }^{26}$, Mentor universel ${ }^{27}$... L'inspiration fénelonienne de l'immense production pédagogique de la deuxième moitié du $\mathrm{XVIII}^{\mathrm{e}}$ siècle, n'est plus à démontrer.

12 Toutefois, à la veille de la Révolution, cette " œuvre moderne le plus souvent publiée $e^{28}$ » que sont les Aventures de Télémaque fait l'objet d'une réception doublement «paradoxale ${ }^{29}$ ".

13 De 1715 à 1815, les continuateurs de Fénelon se trouvent autant du côté des philosophes que de celui des «ennemis des philosophes ${ }^{30}$ »- même si ces catégories sont perméables ${ }^{31}$. «Rousseau et Mme de Genlis [lui] ont vraisemblablement dû une part de leur propre succès ", rappelle Albert Chérel ${ }^{32}$, citant à la fois Rousseau et une écrivaine plutôt anti-lumières ${ }^{33}$, prise à partie par d'Alembert, Grimm, Marmontel ou La Harpe. Le patronage de Fénelon est donc paradoxalement revendiqué sur des fronts opposés.

Du coup, à l'époque, mentionner le prélat se fait toujours en précisant les contours stricts de ce que l'on retient de lui. Les titres cités plus haut le suggèrent. Les épithètes mélioratives - "Véritable ", « vertueux », « universel » - suggèrent que la mention du double fictionnel des Aventures de Télémaque nécessite un éclaircissement quant à l'interprétation que l'on en fait. 

fénelonienne - la polémique et le génie poétique - afin de préciser qu'il ne retient que le second. Fénelon a été " persécuté pour des disputes aujourd'hui méprisées », écrit le pourfendeur du fanatisme, se débarrassant de la réception théologique des œuvres du prélat. En revanche, quand il célèbre l'auteur de ces « ouvrages de prose dans lesquels on a le mieux imité le style de Racine, [avec] cette pureté qui seule met le génie dans tout son jour, et sans laquelle le génie ne déploierait qu'une force monstrueuse ", Voltaire récupère habilement ce que le parti catholique n'a pas su reconnaître en son temps : la «prose tant poétique » qui a suscité le «dégoût» de Nicolas Gueudeville ${ }^{34}$, «poétiquement outré[e] » pour Gaston de Noailles, évêque de Châlons ${ }^{35}$, et ces passages « outrés » que le chanoine Phélipeaux, proche de Bossuet, annote dans L'Explication des Maximes des saints ${ }^{36}$. En outre, par le syntagme «force monstrueuse » Voltaire pourrait bien viser les tenants du « roman antiphilosophique » comme code de moralité.

Sur le front antiphilosophique, la position à tenir sur Fénelon est plus délicate Comment ne pas reprendre à son compte l'apologue catholique le plus efficace, si l'on en croit les portraits avant et après le préceptorat de Fénelon du duc de Bourgogne par Saint-Simon? Néanmoins, le patronage d'un auteur visé par une condamnation papale et une disgrâce à Cambrai soulève des réticences. Dans ce contexte, Madame de Genlis évoque paradoxalement Fénelon. Elle critique souvent son style - tout en citant ses supporteurs $^{37}$, en reconnaissant que le Télémaque est un des « ouvrages qui ont le plus influé sur les mœur ${ }^{38}$ ", et en étant débitrice de nombre de ses idées et de sa figure de bon pédagogue ${ }^{39}$. Au tome $\mathrm{V}$ des Américaines, Marie Leprince de Beaumont place ses personnages en funambules rhétoriques. Miss Dorothée et Madame Bonne sont chargées de dire tout à la fois l'excellence du Télémaque et les « erreurs » de l'Explication des Maximes des saints, comme si les visées théologiques, pédagogiques, et même stylistiques de ces deux ouvrages étaient séparables ${ }^{40}$. «Mme de Beaumont emprunte à Fénelon le procédé de la mise en récit ou en action dialoguée des enseignements ${ }^{41}$ ", mais c'est en héritière à demi du prélat que cette femme apologiste se place.

Ainsi, la réception de Fénelon fait l'objet d'une séparation de ce qui pourtant, dans son œuvre, est intimement mêlé : génie poétique pour les uns, plus souvent les philosophes (Voltaire), mais pas uniquement (Marie Leprince de Beaumont), efficace pédagogue pour les autres, plus souvent défenseur de la foi (Madame de Genlis, par exemple). Encore en 2015, avec Le Sourire de Mentor ${ }^{42}$, Benedetta Papasogli suggère le même schisme chez les lecteurs de Fénelon. D'un côté, on aurait les interprètes d'un « robuste enracinement de l'œuvre de Fénelon dans une fonction pédagogique toute orientée à intercepter les exigences de l'histoire, et à agir sur le réel» - qu'elle associe aux travaux de François-Xavier Cuche. De l'autre, les travaux de Françoise Berlan et de Thomas Pavel révèleraient, selon elle, une «distanciation du rapport entre la représentation et son objet, voire entre le livre et le réel ${ }^{43} \%$. Selon la spécialiste italienne, ce paradoxe continué de la réception fénelonienne provient du fait que le statut du «livre ", ses visées « imaginaires » et "réelles » est pétri "d'ambivalences » chez Fénelon, tout comme son rapport à l'image ${ }^{44}$. Comme ce prénom bifrons, "Mentor", nom commun et nom propre à la fois, le poétique et le rhétorique, l'écriture fictionnelle et l'art oratoire sont intimement tissés dans l'apologue fénelonien.

Dès 1786, lorsqu'un roman antiphilosophique puise dans le fonds désormais commun des descriptions et enseignements célèbres du Télémaque, c'est pour détisser le subtil 
tissage de morale et de romanesque et mettre en images la voie de la stricte orthodoxie, de la façon la plus vive et la plus immédiatement efficace possible.

\section{Descriptions et enseignements féneloniens dans Cruzamante}

Comme de nombreux autres apologues de l'époque, la visée première de Cruzamante pourrait bien être de « retourner contre les Lumières antichrétiennes les séductions littéraires que leurs partisans ne cessent de peaufiner ${ }^{45}$ ». Pourtant, dans le domaine strictement littéraire, Marie-Françoise Loquet ne fait preuve d'aucune inventivité. Fabrice Preyat a bien démontré à quel point Cruzamante se nourrissait en grandes parts du Pèlerinage de Colombelle et Volontairette vers leur bien-aimé dans Jérusalem ${ }^{46}$, des livres d'emblèmes du bénédictin Benoît Van Haeften ${ }^{47}$ (où Cruzamante rejoue en espagnol "Staurophile ", stauros signifiant croix en grec ${ }^{48}$ ), de la Peregrinación de Philotea al Santo Templo y Monte de la $\mathrm{Cruz}^{49}$ et du Camino Real de la $\mathrm{Cruz}^{50}$. Le chercheur note à cet égard que «les œuvres quiétistes ont certainement valeur de transmission dans ces courants $^{51}$ ». Au-delà de cette valeur de transmission, les fables du quiétiste Fénelon mettent à disposition de Loquet une base de données qu'elle importe directement dans sa fiction narrative.

De façon attendue, Marie-Françoise Loquet reprend à Fénelon le matériel idéologiquement licite du Télémaque. En premier lieu, ce sont les expressions directes d'une morale chrétienne orthodoxe qui favorise les pauvres : «Plus heureuses que des Impératrices, nous n'avons rien, et nous possédons tout " disent les compagnes de Cruzamante telles les ombres du Télémaque qui «ne veulent plus rien; ont tout sans rien avoir ${ }^{52}$ ». Comme chez Fénelon, la morale chrétienne n'achoppe que sur le découragement: "L'unique grâce que je vous demande, c'est de me secourir contre le Découragement, ce furieux ennemi, qui me poursuit avec tant d'acharnement ${ }^{53}$ » dit Cruzamante à l'« Amour-souffrant » ainsi que Fénelon dirigeait Madame de Maintenon : "Supportez tout, supportez-vous vous-mêmes dans vos découragements. Ô qu'on a besoin de patience avec soi. Souvent on se décourage de s'être découragé. Au contraire, qui ne se décourage jamais de ses découragements les tourne à profit ${ }^{54}$ ». On retrouve également dans Cruzamante, pour reprendre la formule de François-Xavier Cuche, l'expression d'une «pensée sociale catholique ${ }^{55}$ ». Le modèle de concorde opposé à la "Propriété ", que Cruzamante rencontre sur son chemin, reprend celui des cités idéales (Bétique, Salente) du Télémaque : «Cette femme est la Propriété ; défiez-vous de ses ruses, elle est la perte des âmes qui vivent en communauté. [...] N'ayez rien à vous, que tout soit en commun, votre état doit représenter celui de l'Église naissante. Soyez indifférentes à ce qu'on laisse à votre usage ${ }^{56}$ ". N'y a-t-il ici qu'un simple héritage de valeurs et principes chrétiens à un siècle de différence?

21 Les descriptions qui émaillent le pèlerinage de Cruzamante et de ses compagnonnes indiquent un héritage plus précis entre le quiétiste et la femme antiphilosophe. Elles empruntent directement leur matériau lexical aux fables rédigées pour le duc de Bourgogne par Fénelon. Par exemple, l'arrivée de «L'Esprit du monde » est l'occasion d'un collage de la description de la grotte de Calypso dans le Télémaque et du jardin de Crésus dans L'Anneau de Gygès :

Partout l'Esprit du monde a des châteaux, des palais, et même des temples; il les change à son gré de décorations et d'ornements, selon l'âge et la condition des 
personnes, qu'il veut séduire. À quelque distance de ce séjour enchanteur, où ce trompeur avait triomphé de l'infidèle compagne de Cruzamante, il avait fait dresser un pavillon, dont la riche étoffe était soutenue avec art, par des agrafes de diamants, entourées de rubis et de topaze, qui jetaient une lumière éblouissante. De doux zéphyrs y entretenaient la fraîcheur, et les rosiers, les jasmins, les orangers y répandaient à l'envi leur agréable odeur. On ne pouvait y arriver qu'en marchant sur un gazon, émaillé de fleurs, et bordé de cascades, qui précipitaient leurs eaux argentines avec fracas ${ }^{57}$.

On arrive dans cette maison dont tous les murs étaient de jaspe ; le toit était de cuivre fin et brillant comme l'or: les lits étaient d'argent, et tout le reste des meubles de même: tout était orné de diamants et de pierres précieuses. Tout le palais était sans cesse rempli des plus doux parfums; et pour les rendre plus agréables, on en répandait de nouveaux à chaque heure du jour. Tout ce qui servait à la personne du Roi était d'or ${ }^{58}$.

[...] mille fleurs naissantes émaillaient les tapis verts dont la grotte était environnée. Là on trouvait un bois de ces arbres touffus qui portent des pommes d'or, et dont la fleur, qui se renouvelle dans toutes les saisons, répand le plus doux de tous les parfums; ce bois semblait couronner ces belles prairies, et formait une nuit que les rayons du soleil ne pouvaient percer. Là on n'entendait jamais que le chant des oiseaux, ou le bruit d'un ruisseau, qui, se précipitant du haut d'un rocher, tombait à gros bouillons pleins d'écume, et s'enfuyait au travers de la prairie ${ }^{59}$.

Par cette démarche, Marie-Françoise Loquet semble signaler à son public - qui ne pouvait manquer de reconnaître les originaux -, que son invention à elle ne se situe pas sur le terrain poétique.

La comparaison des classes lexicales du Télémaque et de Cruzamante grâce au logiciel IRaMuTeQ confirme cette impression. La classification lexicale ou " méthode Reinert » regroupe les termes qui ont tendance à apparaitre dans les mêmes contextes au sein de l'œuvre. Dans le Télémaque, les classes 2, 3, 4 et 5 signalent quatre sources différentes de l'invention romanesque: l'aventure maritime, la quête du père, la guerre et la construction politique. Une seule classe, la première, regroupe le vocabulaire de la moralité et fait spécifiquement apparaître les péchés et les vertus catholiques :

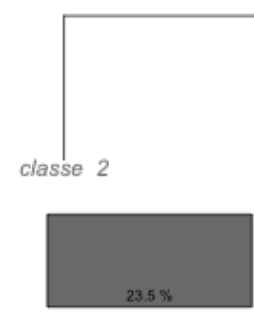

oeil

rocher

onde

couvrir

cri

feu

sombre

couler

bruit

pousser

abîme

noir

flamme

mer

ciel

percer

montagne

vent

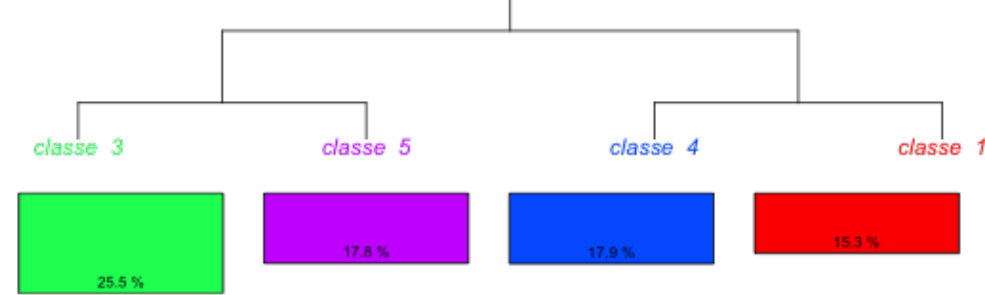

idoménée

allié philoclès

télémaque

timocrate

protésilas

mentor

découvrir

surprendre

adraste

phalante

phénicien

chef

armée

dauniens

renvoyer

camn

peuple
nation
loi
art
ville
cultiver
commerce
travail
pays
moeurs
abondance
paix
établir
nécessaire
laborieux
agriculture
utile
voisin

homme

vertu

gloire

ambition

avarice

punir

plaisir

propre

orgueil

crainte

humain

détester

nature

amour

condamner

solide

grandeur

nécessité 
Dans Cruzamante, au contraire, le lexique romanesque (classes 2, 3, 4 et 5 du Télémaque) n'apparaît presque pas au niveau macrostructural des statistiques, preuve que même les emprunts aux fables féneloniennes n'ont été qu'épisodiques. La classe 3 fournit le cadre minimal de l'inventio, puis les classes 1, 2 et 4 proposent un même champ lexical de la souffrance : la souffrance liée à la faute de la créature même, celle infligée par le créateur, puis celle qui donne accès à l'extase mystique, « l'Amour-souffrant » :

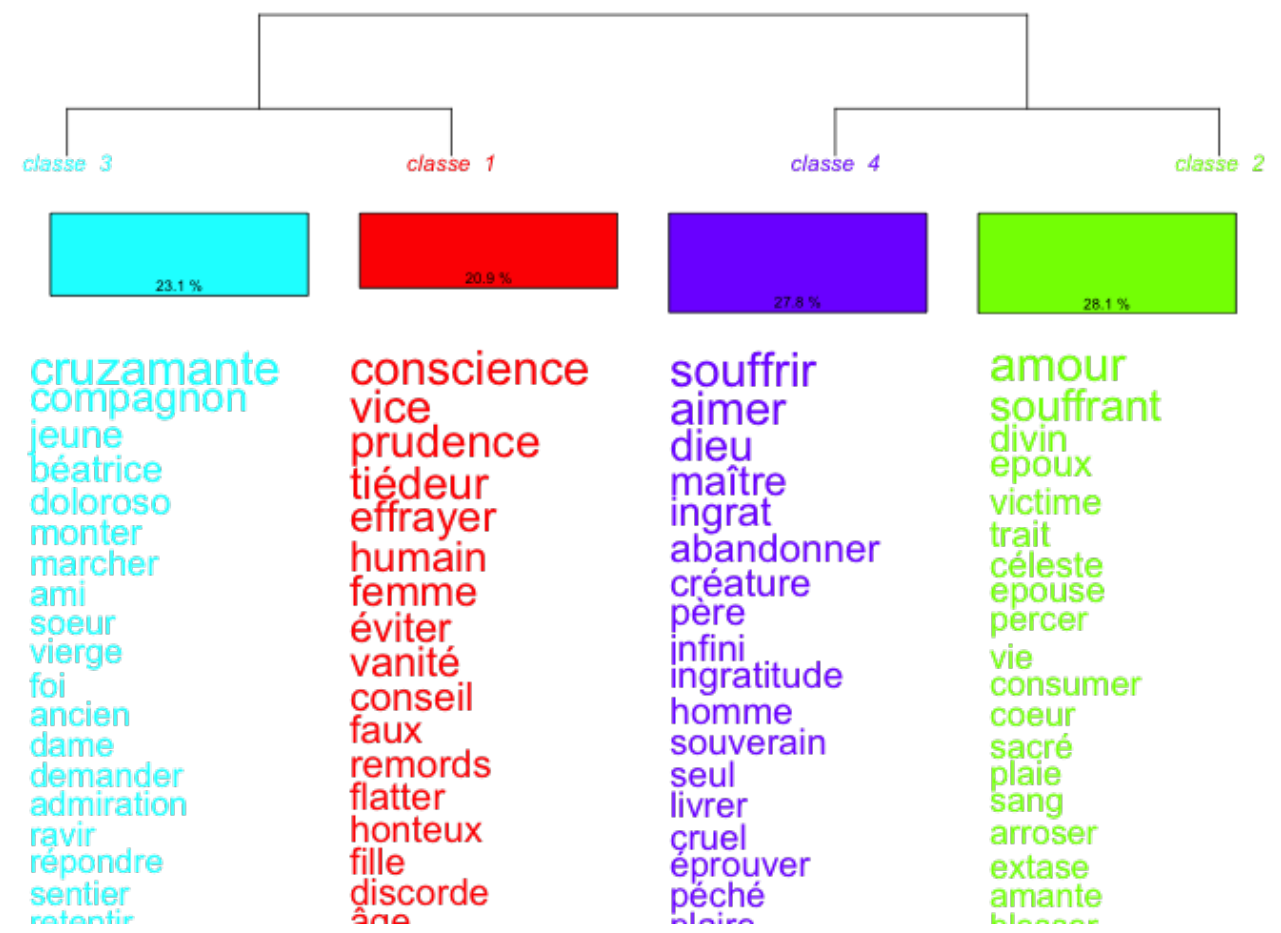

Ainsi, l'étude macroscopique du lexique des deux apologues montre à quel point l'un et l'autre privilégient soit les termes à fort ancrage poétique soit les termes à fort ancrage rhétorique, le champ lexical de la souffrance portant la visée rhétorique principale du roman, tout entier orienté vers la recherche de «l'Amour-souffrant ». Marie-Françoise Loquet avait d'ailleurs expliqué, cinq ans avant la publication de Cruzamante, son parti pris d'un romanesque avant tout rhétorique :

On n'a pas cherché à orner [Le Voyage de Sophie et d'Eulalie] d'images [...] de charmantes descriptions. On néglige tous ces vains ornements; \& c'est avec une espèce de regret qu'ils y trouvent naturellement leur place. On s'y attache surtout aux instructions morales, aux discours simples \& pathétiques, qui font voir, à n'en point douter, qu'on ne souhaite que d'y exposer le langage de la vérité \& la rhétorique du cœur ${ }^{60}$.

Si Fénelon lui-même est le théoricien de la pureté contre les "vains ornements ", la fortune de ses œuvres littéraires suggère un échec de sa propre politique stylistique. Au contraire, Marie-Françoise Loquet demeure en cohérence avec ce projet de n'exposer dans le roman que «le langage de la vérité \& la rhétorique du cœur». Bien plus, ces lignes pourraient bien constituer une opposition explicite à la rhétorique de la «peinture » défendue par Fénelon dans les Dialogues sur l'éloquence ${ }^{61}$.

À partir de cette idée, la femme antiphilosophe reprend à Fénelon une distinction axiologique entre mollesse efféminée et courage ferme, courage dont la rigueur n'est interrompue que par les symptômes indéniables de la véritable "rhétorique du cœur »: les larmes. Ainsi, Cruzamante est une «femme forte» tandis que Staurophile était une femme «faible ${ }^{62} »$; le «courage mâle» de la «s sœur du Comte d'oliva, de la «sainte 
fermeté » et, in fine, de Cruzamante ${ }^{63}$, ne manquent pas de rappeler l'Antiope de Fénelon, « majestueuse et adroite à tirer de l'arc ", dont " les mains ne méprisent pas le travail ", qui «d'un seul regard se fait entendre » et ne craint pas de se mesurer à un sanglier ${ }^{64}$. Participant de cette même axiologie issue du Télémaque, ce qui est «mou ", «faible » et/ou « efféminé » est assimilé à la lâcheté et vivement condamné. Ainsi, la "démarche» de «l'Esprit du monde » est «molle et efféminée ${ }^{65}$ ». Et, comme les personnages du Télémaque, Cruzamante manifeste régulièrement une légitime émotion en « arrosant de ses larmes » son environnement ${ }^{66}$.

Malgré ce cadre théorique et idéologique manifestement emprunté au prélat, la diégèse $\mathrm{du}$ roman suggère une critique de la mystique fénelonienne.

\section{Portrait d'un Fénelon quiétiste sous la « Tiédeur »}

Au chapitre 11 de Cruzamante, la rhétorique condamnatoire de la mollesse efféminée semble se retourner contre son créateur - Fénelon - lorsque le personnage allégorique de la « Tiédeur » entre en scène :

Ses yeux, à demi éteints, jetaient des regards, qui prévenaient en sa faveur. Une blancheur d'albâtre formait son teint, et en avait fait disparaître cette pâleur, qui la défigurerait devant le Seigneur. Une langueur dans sa démarche annonçait la faiblesse de sa santé et le mal critique, dont elle était attaquée. Un air d'indifférence, une certaine grandeur d'âme, une liberté, mêlée de légèreté et de retenue, répandaient sur son visage, un je ne sais quoi qui la rendait intéressante ${ }^{67}$.

Cette description focalisée sur le regard et la pâleur du visage semble réécrire, sous l'angle de la maladie, les premières lignes que Saint-Simon dédie à Fénelon à sa mort :

Ce prélat était un grand homme maigre, bien fait, pâle, avec un grand nez, des yeux dont le feu et l'esprit sortaient comme un torrent, et une physionomie telle que je n'en ai point vu qui y ressemblât, et qui ne se pouvait oublier, quand on ne l'aurait vu qu'une fois. [...] Tous ses portraits sont parlants, sans toutefois avoir pu attraper la justesse de l'harmonie qui frappait dans l'original, et la délicatesse de chaque caractère que ce visage rassemblait ${ }^{68}$.

Bien plus, la mention de l'« indifférence ", notion clef de la doctrine du pur amour, héritée d'Ignace de Loyola et de Saint-François de Sales, et du fameux " je ne sais quoi » des mystiques, renforce l'hypothèse d'une référence au quiétiste sous le portrait de la Tiédeur. Il en va de même, dans la suite, de la " sécheresse » attribuée à ce personnage. Elle ne manque pas de rappeler celle que Fénelon s'attribue de façon récurrente dans sa correspondance avec Madame Guyon.

Ainsi, dans Cruzamante, le faux dévot, figure topique de la rhétorique complotiste du "roman antiphilosophique ${ }^{69}$ " pourrait bien s'appuyer sur la figure réelle du Fénelon quiétiste. La référence semblerait anecdotique, voire non avérée, si un point précis de théologie - la nécessité de pratiquer les vertus théologales de l'espérance et de la charité - ne venait l'étayer. C'est, en effet, sur ce point précis que les attaques de Bossuet et la condamnation papale se sont focalisées.

\subsection{Une rhétorique chrétienne qui replace la Charité en son centre}

Au sujet de la Tiédeur, la narratrice de Cruzamante écrit : "Sa funeste erreur est d'être persuadée, qu'elle obtiendra, sans travail, un royaume, qui ne s'acquiert que par la violence $^{70} »$. La deuxième partie de la citation commente de façon topique un passage 
de l'Évangile selon Saint $\mathrm{Luc}^{71}$. Fénelon a également pu le faire ( Ce n'est que par violence qu'on entre dans le royaume de $\left.\operatorname{Dieu}^{72} »\right)$. Le groupe prépositionnel «sans travail », lui, cible de façon plus originale un point d'opposition réel entre Fénelon et ses opposants : la pratique de la charité, ou œuvres, pour assurer son salut.

En effet, dans la mystique de Fénelon héritée de Saint François de Sales ${ }^{73}$, de Saint Bernard et de Saint Clément d'Alexandrie ${ }^{74}$, le croyant, défait de l'amour servile, puis de l'amour sans charité, dépasse les échelons de l'amour d'espérance et de l'amour de charité pour atteindre le "pur amour ", c'est-à-dire l'amour le plus désintéressé qui soit pour Dieu. Dans cette exigence radicale, Bossuet, puis le pape, identifient une hétérodoxie : évincer deux des trois vertus théologales, l'espérance (dans le salut) et la charité (les œuvres qui donnent accès au salut), au profit de la « pure foi ».

Dans cet ordre d'idées, la Tiédeur est l'adversaire le plus redoutable de la « Religion » et de la «Sainte fermeté" dans Cruzamante. Elle séduit presque la moitié des compagnonnes de l'héroïne (17 sur 35 ) en proposant de remplacer les «exercices spirituels » et «directeurs outrés, qui sont si minutieux, qui damnent tout le monde » par des abstraites « obligations [...] plus essentielles ${ }^{75} »$.

À l'inverse, la narration du roman jusqu'à l'apothéose de Cruzamante sur le MonteDoloroso répète à l'envi la nécessité d'agir pour garantir le salut. Les "œuvres", qualifiées de «bonnes œuvres » ou d'« œuvres satisfactoires » reviennent dix-sept fois. Ce sont ces "tribulations", et non une "oraison perpétuelle», qui donnent accès à la purification et donc à l'Union en Dieu : « Pour te purifier et te rendre plus conforme à ton Bien-aimé, tu dois encore passer par le creuset de nouvelles tribulations ${ }^{76}$ ", explique l'« Amour-souffrant » à Cruzamante.

Bien plus, lorsque le roman met en scène une vision du Pur Amour, c'est l'allégorie de la Charité qui se trouve en son centre : «La Religion, pour prouver à ses jeunes disciples la vérité de ses paroles les éclaira d'une nouvelle lumière, les fit pénétrer dans les cœurs de Cruzamante \& de Béatrice. Quel fut leur étonnement! lorsqu'elles virent que la Charité agissait en Souveraine dans ces cœurs, qu'elle y détruisait, qu'elle y renversait, qu'elle $\mathrm{y}$ arrachait, sans pitié, tout ce qui s'opposait au règne du Pur Amour ». Ainsi, Marie-Françoise Loquet propose à ses lectrices un itinéraire mystique bien éloigné de celui que les critiques ont décelé dans le Télémaque : le romanesque - au premier chef desquels le plaisir des descriptions - en est évacué et l'excipit substitue à la transfiguration lumineuse et éthérée de Mentor en Minerve les actions concrètes et violentes de la Charité.

D'ailleurs, l'« Amour-souffrant ", avec ses 134 occurrences, est mis en lieu et place du pur amour fénelonien qui n'est cité que trois fois de manière conventionnelle. C'est lui qui est promis à «l'épouse » du Cantique des cantiques et c'est avec lui que Cruzamante s'unit en mourant à la fin du roman. À «l'Amour-souffrant» qu'elle poursuit sans relâche, l'héroïne déplore : «Si mon amour pour vous avait été plus pur, plus agissant, je jouirais encore de votre sainte préférence ${ }^{77} »$. C'est donc au prix d'une inflexion du texte biblique que l'« époux » ou «bien-aimé » entre en conformité avec une théologie qui replace l'agir ou les « œuvres » en première ligne de la mystique chrétienne.

36 Ce repositionnement n'est pas qu'une façon de rétablir l'orthodoxie de la mystique chrétienne. Elle est entre aussi en adéquation avec l'urgence politique et les critères esthétiques de son époque. 


\subsection{Rebranding mystique à la veille de la Révolution : « l'Amour souffrant »}

besoin, la visée violemment combative du roman antiphilosophique pour s'« impose[r] aux partisans du siècle ${ }^{79}$ ». Dans la même perspective, au tome $\mathrm{V}$ des Américaines Miss Dorothée et Mademoiselle Bonne citent le cas Fénelon. Elles s'adressent à un calviniste pour le ramener à la foi catholique :

Miss Dorothée - Permettez-moi, ma Bonne, de donner un exemple récent qui m'a extrêmement frappée. L'auteur du Télémaque, ce livre que nous aimons tant, avait fait un ouvrage de spiritualitế $e^{80}$ qui renfermait des erreurs. Après maintes et maintes disputes sur ce livre, il fut porté à Rome, où M. de Cambrai était fort aimé. Le pape nomma une assemblée de cardinaux et de docteurs pour examiner l'ouvrage ; et malgré le bien qu'il voulait à l'auteur, le livre fut condamné. On reçut cette condamnation en France, où l'auteur brûla lui-même son ouvrage et excommunia ceux qui le garderaient: sa soumission, dans laquelle j'ai trouvé un vrai héroïsme, apaisa tout.

Madame Bonne - Si M. de Cambrai se fut révolté et qu'il eut porté ses erreurs dans d'autres royaumes, il aurait été retranché de l'Église, on eut notifié sa condamnation et sa cause à toute l'Église; et si le plus grand nombre des évêques eut acquiescé à cette condamnation ou formellement ou tacitement, il aurait été condamné comme dans un concile. Pouvez-vous nier, Monsieur, que l'Église ne se soit servie inviolablement de la même méthode quand elle n'a pu s'assembler?

Le calviniste - Quand je vous l'avouerais, Mademoiselle, à quoi cela servirait il ?

Madame Bonne - À prouver l'uniformité que l'Église a toujours observée dans la condamnation des hérétiques ${ }^{81}$.

Dans cet échange, l'Église se voit drapée d'un trait de rigueur morale en chassant, en son sein même, ses ennemis intérieurs, tandis que le repentir suggère la possibilité de conversion aux ennemis extérieurs ici directement visés: Fénelon ne s'est pas "révolté ", il n'a pas "porté ses erreurs dans d'autres royaumes", il a fait acte de «soumission». Bien plus et au-delà de cette rhétorique antiphilosophique traditionnelle, c'est une esthétique qui valorise étonnamment la violence que la mention de Fénelon permet : il «brûla lui-même son ouvrage et excommunia ceux qui le garderaient » et, par ces gestes, il « apaisa tout ».

Cette valorisation morale de la violence, typique de l'époque, prend la forme, dans Cruzamante, de réécritures étonnantes du texte biblique et de la tradition mystique. Le père qui abandonne son fils dans les Évangiles ${ }^{82}$ devient le père qui bat puis console sa fille : «Soumettez-vous donc en tout à votre Dieu; baisez la main d'un Père, qui vous frappe, pour vous consoler ensuite avec plus d'abondance ${ }^{83} »$. Ailleurs, la contemplation des stigmates réservée aux âmes mystiques prend le tour d'une délectation sanguinaire encore inédite dans les ouvrages mystiques :

L'Amour-souffrant, content de sa générosité, lui laissa considérer à son aise les blessures sans nombre, que nos crimes lui ont attirées. Cette sainte Amante les regardait avec une affection, pleine d'ardeur, et elle recueillait avec transport le sang qui en coulait de toutes parts; toute couverte de ce sang adorable, elle ne pouvait se lasser de contempler le digne Objet de son amour; et plus elle le contemplait, plus elle désirait de lui devenir semblable ${ }^{84}$.

Traditionnellement une étape de la vie contemplative vers le plaisir de l'Union mystique, les souffrances semblent ici être recherchées per se par les jeunes filles :

La Religion fit apercevoir à ses disciples un sentier, presque tout couvert de cailloux pointus, et bordé par une haie d'épines, elle les avertit qu'il fallait absolument 
passer par ce sentier des douleurs et des combats. À cette nouvelle, Cruzamante ne put se contenir. ' $O$ voie délicieuse! (s'écria-t-elle) ô chemin plein de charmes! Recevez-moi et me conduisez à mon Epoux souffrant' ! En disant ces mots, elle courut dans cette laborieuse carrière, avec une vivacité incroyable. Bientôt, les pierres aiguës entrèrent dans ses pieds, et furent teintes de son sang; mais cette sainte Amante de Jésus crucifié n'y fit point la moindre attention, et ne s'exprima que par des élans d'amour. Béatrice, ravie du courage de sa chère sœur l'admira et la suivit avec une fermeté sans égale : toutes les autres s'empressèrent à l'envi de marcher sur leurs pas ${ }^{85}$.

Cette esthétique atteint son apogée dans la mort de l'héroïne :

Pendant sa vie mortelle, cet amour l'épuisa de fatigues \& de travaux; enfin le moment étant arrivé, où l'holocauste devait être tout consumé le même amour fit tomber, dans la plus terrible des agonies, cette adorable Victime. C'est cet amour, qui le chargea du poids énorme de sa croix \& qui l'entraîna, par une force victorieuse, vers les plus horribles supplices. C'est cet amour, qui réduisit sa chair en lambeaux, par une cruelle flagellation, qui couvrit de crachats son adorable visage, qui couronna sa tête d'épines, qui perça ses pieds \& ses mains, qui lui ouvrit le côté, pour t'y ménager, ô ma chère Épouse, un asile contre toutes les fureurs de tes ennemis ${ }^{86}$.

De façon attendue depuis l'incipit, la mort de Cruzamante réalise dans les hyperboles et superlatifs le terme de cette croissance corrélative de la souffrance (« la plus terrible des agonies", «les plus horribles supplices») et de la gloire («adorable», littéralement, que l'on peut adorer, comme une sainte). Mais ce qui frappe, ce sont à la fois la violence et la trivialité de certains des termes choisis. Les «lambeaux » font traditionnellement référence aux reliques des saints, et la «flagellation» et "couronne[e] d'épines», aux pieds, mains et côté percés à la Passion de Jésus. En revanche, l'épithète «cruelle » et les « crachats » manifestent un écart par rapport au texte biblique et à la tradition. Bien plus, « Après sa mort, [...] Ses cendres étaient pour ces filles, comme une source de vertus, et un fer toujours brûlant, où elles allaient chaque jour puiser une nouvelle ardeur pour leur Bien-aimé ${ }^{87} »$, nous dit l'auteure de Cruzamante, réalisant une nouvelle alliance du désir ( ardeur ») et de la violence ( un fer toujours brûlant»). Fabrice Preyat rappelle combien Marie-Françoise Loquet, comme les autres femmes apologistes, est sensible aux effets de mode afin d'élargir et de séduire son audience et de convaincre les incrédules. Elle tire "profit des modes littéraires alimentées par ses contemporains ", ses œuvres s' « arrime[nt] à la longue suite des genres en vogue sous les Lumières ${ }^{88}$ » et Cruzamante a été lue comme une " hyper-Justine ${ }^{89}$ » en référence à Sade.

41 Ainsi, paradoxalement, tout en rétablissant l'orthodoxie de la mystique par le primat de l'agir, la violente pédagogie mortifère ${ }^{90}$ de Cruzamante s'accorde à la violence du climat politique, et à l'esthétique des années 1780 , romans libertins et esthétique sadienne inclus. C'est en cela, qu'elle s'éloigne le plus du modèle fénelonien et qu'elle produit, sans doute, une autre forme d'hétérodoxie.

\section{Conclusion : Cruzamante, un manifeste rhétorique de la « sainte fermeté » contre l'insinuatio}

Dans Cruzamante ou la sainte amante de la croix, Marie-Françoise Loquet reprend superficiellement les célèbres descriptions et contenus théologiques non problématiques des œuvres de Fénelon. En profondeur, ce rapport au modèle se révèle 
paradoxal, tant la rhétorique semble dirigée contre le quiétisme et son roman pédagogique le plus célèbre, Les Aventures de Télémaque. Le personnage central de la Tiédeur le suggère : c'est moins contre le danger de la philosophie que contre celui de la douceur que la jeune femme de 37 ans met en garde ses jeunes compatriotes : «la Tiédeur, qui les enchante par ses caresses et sa langue de serpent, s'est entièrement rendue maitresse de leurs cœurs, et elle les conduit, par des routes détournées, dans le plus affreux des précipices ${ }^{91} »$. Par l'image du « serpent » et l'idée de détour (circumitio), Marie-Françoise Loquet se distingue explicitement de l'insinuatio ${ }^{92} \mathrm{du}$ prélat et précepteur de 49 ans, Fénelon ${ }^{93}$. Sa rhétorique qui prône à l'envi la "sainte fermeté " s'effectue en ligne droite, jusqu'à évacuer le romanesque même du roman et multiplier les subordonnées consécutives pour répéter la corrélation positive entre souffrance terrestre et gloire divine : «Plus vos combats seront violents, plus le secours d'en haut sera puissant ; plus votre croix sera parfaite, plus vous serez agréables à Jésus-Christ ; enfin, plus vous souffrirez sur la terre, plus votre gloire sera grande dans le Ciel ${ }^{94}$ ", explique Cruzamante aux jeunes femmes qui la suivent. « Nous ne voulons avec lui, ni paix, ni réconciliation, plus il nous haïra, plus nous nous réjouirons ${ }^{95} "$, renchérissent les jeunes femmes dans cette traversée du désert romanesque où l'« Amour-souffrant " est la Terre Promise :

- Que voulez-vous, digne Objet de mon cœur? venez-vous briser mes liens, ou augmenter mes douleurs ? Souffrir ou mourir, voilà où tendent tous mes vœux !

- Ma Bien-aimée, (reprit l'Amour-souffrant) ce moment, où ton âme s'envolera dans le sein de ton Époux, n'est pas encore arrivé ; mais console-toi tu n'es pas au bout de tes souffrances ${ }^{96}$.

Cette clarté et cette pauvreté stylistiques sont aussi géographiques et logistiques. À l'errance aventurière de Télémaque dans la mer Méditerranée se substitue une trajectoire rectiligne vers une destination anticipée pour un projet précisément et aisément établi : bâtir un monastère dans un lieu escarpé, que Cruzamante prend soin d'acquérir avant de se mettre en route et pour lequel elle commande une « exécution prompte » à un " architecte $^{97}$ »!

- Ô mon Amour, où voulez-vous que je vous suive? demande Cruzamante à Dieu. Sur le Monte-Doloroso, (répliqua clairement le Seigneur) c'est sur cette montagne de douleurs, que je veux conduire tes pas, et éprouver ta fidélitées.

Ainsi, les prises de distance narratives, théologiques et lexicales avec la rhétorique fénelonienne laissent entrevoir, in fine, une opposition plus profonde entre une stylistique chrétienne rectiligne et l'insinuation fénelonienne dont les marques énonciatives mériteraient sans doute une étude dédiée.

\section{NOTES}

1. R. Girard, La Violence et le sacré, Paris [Grasset, 1972], Fayard/Pluriel, 2010, p. 412.

2. D. M. Mcmahon, "The real counter-enlightment: the case of France", dans Isaiah Berlin's Counter-Enlightment, American Philosophical Society, Vol. 93, Part 5, edited by J. Mali and R. Wokler, p. 91-104 (ici p. 100). 
3. D. Masseau, Les Ennemis des philosophes, Paris, Albin Michel, 2000, p. 20.

4. J.-P. Migne, Collection intégrale et universelle des orateurs sacrés, 99 volumes (Paris, 1844-1846), volume 65, CEuvres complètes de Cambacérès, Sermon III, « Sur les incrédules », p. 1047-1048.

5. D. M. Mcmahon, op. cit., p. 94.

6. D. Masseau, «Qu'est-ce que les anti-Lumières ? ", Dix-huitième siècle, 2014/1, n 46, p. 107-123.

7. N. Brucker, «Élie Fréron, promoteur du roman antiphilosophique», dans S. Barthélemy,

A. Cariou et J. Balcou dir., Élie Fréron, Polémiste et critique d'art, Rennes, PUR, 2001, p. 87-96.

8. B. Papasogli, "Pour et contre la lecture: Paradoxes du Télémaque ", Littératures Classiques, 2010, n 70, p. 315-330 ; voir aussi B. Papasogli, Le Sourire de Mentor, Paris, H. Champion, 2015, chapitre IX, «Les Ambivalences du livre », p. 213-225.

9. N. Brucker, op. cit., p. 89.

10. Ibid., p. 95.

11. C'est sans doute un trop grand pouvoir conféré à la fiction au détriment de la théologie qui expliquerait le rejet ecclésiastique des œuvres de Marie Huber. Y. Krumenacker, «L’Usage de la fiction dans l'apologétique de Marie Huber », dans F. Preyat dir., Femmes des anti-lumières, femmes apologistes, Bruxelles, Éditions de l'université de Bruxelles, 2016, p. 70.

12. Dans sa préface aux Contes moraux (1773), elle feint de reprendre à son compte les critiques qui lui sont adressées : «Encore si son style compensait l'ennuyeux des leçons qu'elle débite, on pourrait les lui passer : mais ce style est maudit; Fréron nous en avait avertis il y a plus de six ans ». Voir J. M. Leprince de Beaumont, Contes moraux, "Préface», dans Contes et autres écrits, éd. B. Kaltz, Oxford, Voltaire Foundation, 2000, p. 159.

13. On pense aux développements sur le fini et le borné humains face à l'infini divin qui sont directement issus de l'ouvrage fénelonien.

14. J. M. Leprince de Beaumont, Les Américaines ou les preuves de la Religion chrétienne par les lumières naturelles, Lyon, 1770, tome I, p. 47 et 73-74, et tome II, p. 101.

15. Voir par exemple l'échange entre La Bonne et Lady Spirituelle dédiée au syllogisme. J. M. Leprince de Beaumont, Les Américaines, ibid., tome II, p. 30.

16. « Histoire de Léontine », tome I, « Histoire de Mélicourt », tome II et « Histoire de Bervile », tome V.

17. F. Preyat, " LOQUET, MARIE-FRANÇOISE (1750-18..)", dans Dictionnaire des anti-lumières et des antiphilosophes, France 1715-1815, dir. D. Masseau, tome II, J-Z, p. 987.

18. M.-F. Loquet, Cruzamante, ou la sainte amante de la Croix, Paris, Morin, 1786, p. 7-8.

19. Ibid., p. 9.

20. Ibid., p. 2.

21. R. Girard, op. cit., p. 383 et 400.

22. M.-F. Loquet, op. cit., p. 244 et p. 448.

23. A. Barruel, Les Heviennes ou Lettres provinciales philosophiques, 1781, volume IV, p. 156-157.

24. L. Rustighi, «Figures de la féminité chez Marie-Françoise Loquet. Un dialogue avec Rousseau ", dans Études sur le $18^{e}$ siècle, Femmes des anti-lumières, femmes apologistes, op. cit., p. 87.

25. L.-A. de Caraccioli, Le Véritable Mentor ou l'éducation de la noblesse, Breslau, 1756.

26. L.-P. Bérenger, Le Mentor vertueux, moraliste et bienfaisant, ou Choix de faits mémorables, d'anecdotes intéressantes, d'entretiens moraux, de lettres et de descriptions propres à inspirer le goût des choses honnêtes et à former le style et le langage des jeunes gens, Paris, chez Nyon l'aîné, 1788.

27. Journal d'éducation fondé par l'abbé Roy en 1784.

28. F.-X. Cuche et J. Le Brun dir., « Avant-propos », Fénelon. Mystique et politique (1699-1999), actes du colloque international de Strasbourg pour le troisième centenaire de la publication du Télémaque et la condamnation des Maximes des Saints, Paris, Champion, 2004, p. 9.

29. F.-X. Cuche, «La morale dans les ouvrages pédagogiques de Fénelon », dans Nouvel état présent des études sur Fénelon, Amsterdam / Atlanta, Rodopi, 2000, p. 59-60.

30. D. Masseau, Les Ennemis des philosophes, op. cit. 
31. Yves Krumenacker remarque subtilement qu'une auteure comme Marie Hubert détient une entrée et dans le Dictionnaire des femmes des Lumières de V. André et H. Krief, et dans le Dictionnaire des anti-Lumières de Didier Masseau. Y. Krumenacker, "L'Usage de la fiction dans l'apologétique de Marie Huber », op. cit., p. 62.

32. A. Chérel, Fénelon au XVIII siècle en France (1715-1820). Son prestige. Son influence. Tableaux bibliographiques (supplément), Paris [Hachette, 1917], Genève, Slatkine Reprints, 1970, p. 569.

33. Voir F. Preyat dir., Femmes des anti-lumières, femmes apologistes, op. cit.

34. N. Gueudeville, Critique générale des Aventures de Télémaque, chez les héritiers de P. Marteau, Cologne, 1700, p. 39.

35. En note d'une lettre de Bossuet sur le style du Télémaque, Correspondance de Bossuet, nouvelle édition augmentée de lettres inédites et publiée avec des notes et des appendices sous le patronage de l'Académie française, par Ch. Urbain et E. Levesque, tome XII (mai 1699 - décembre 1700), Paris, Librairie Hachette, 1920, p. 6.

36. J. Le Brun, «La spiritualité de Fénelon jugée par un proche de Bossuet, le chanoine Phélipeaux », communication du 19 juin 2015 au colloque " Fénelon » organisé à Strasbourg par Béatrice Guion et François-Xavier Cuche.

37. Mme de Genlis, La Religion considérée comme l'unique base du bonheur et de la véritable philosophie, Paris, 1787, p. 399.

38. Mme de Genlis, Les Veillées du château, Paris, 1784, tome I, p. VIII-IX.

39. «Souvenez-vous de l'histoire de Fénelon et de son élève le duc de Bourgogne ; ils furent à peu près séparés ainsi... Ce jeune prince sentit vivement son malheur ; il aima Fénelon toute sa vie ", Mme de Genlis, lettre de 1791 à Mademoiselle d'Orléans dont on vient de lui retirer la direction, Mémoires, V, p. 142, cité par A. Chérel, Fénelon au XVIII siècle en France (1715-1820), op. cit., p. 471.

40. M. Leprince de Beaumont, Les Américaines, op. cit., tome V, p. 64.

41. A. Chérel, op. cit., p. 569.

42. B. Papasogli, Le Sourire de Mentor, op. cit.

43. Ibid., p. 227.

44. Voir le numéro spécial des Cahiers du GADGES dirigé par Olivier Leplatre et intitulé Le Clairobscur du visible. Fénelon et l'image » (Cahiers du GADGES vol. 14, 2017).

45. F. Preyat, « LOQUET, MARIE-FRANÇOISE (1750-18..) », dans op. cit., p. 987.

46. Boetius Adam Bolswert, Anvers, 1627.

47. 1635 et 1640 .

48. F. Preyat, op. cit., p. 990.

49. Juan de Palafox, 1657.

50. Martín de Herce, 1721.

51. Ibid.

52. M.-F. Loquet, op. cit., p. 343.

53. Ibid., p. 421.

54. Lettre 100. À Madame de Maintenon. Vers février 1690, Correspondance de Fénelon, édition de Jean Orcibal (tomes I-V), Paris, Klincksieck, 1972- 1976, tome II, p.150. Nous soulignons.

55. F.-X. Cuche, Une Pensée sociale catholique. Fleury, La Bruyère, Fénelon, Paris, Cerf, 1991.

56. M.-F. Loquet, op. cit., p. 340-341.

57. Ibid., p. 159-160.

58. Fénelon, Fables, VII, «L'anneau de Gygès ", CEuvres complètes de Fénelon, édition dite de Paris, ou de Saint-Sulpice, ou des quatre éditeurs (Leroux, Gaume [Paris], Lefort [Lille], OutheninChalandre [Belfort]), dix volumes, 1848-1852, "par les soins de MM. Gosselin et Caron" (à présent $O C$ ), tome VI, p. 204.

59. Fénelon, Les Aventures de Télémaque, fils d'Ulysse, éd. M. Haillant, Paris, Nizet, 1993, p. 237-238.

60. M.-F. Loquet, Le Voyage de Sophie et d'Eulalie au palais du Vrai Bonheur, ouvrage pour servir de guide dans les voies du salut, Paris, Berton, 1781, p. 4-5. 
61. Sur ce point, lire J.-P. Grosperrin, «Eloquence et pensée religieuse : la notion de 'peinture' dans Fénelon », L'Information littéraire, mai-juin 1995, p. 19-26.

62. Ibid.

63. M.-F. Loquet, Cruzamante, op. cit., p. 9, 169 et 409.

64. Fénelon, Les Aventures de Télémaque, fils d'Ulysse, éd. M. Haillant, Paris, Librairie Nizet, 1993, p. 573-574 et 582 .

65. M.-F. Loquet, Cruzamante, op. cit., p. 149.

66. Par exemple, M.-F. Loquet, Cruzamante, op. cit., p. 436.

67. Ibid.., p. 129.

68. Saint-Simon, Mémoires, Additions au Journal de Dangeau, éd. Y.Coirault, Paris, Gallimard, 1985, t. V, p. 148.

69. N. Brucker, op. cit., p. 94 et D. Masseau, op.cit., p. 19 : «Un janséniste, l'abbé Gaultier, est un des premiers à évoquer l'idée d'un complot fomenté par les ennemis de la religion, que ceux-ci soient déistes, théistes ou résolument athées ». Note de l'auteur : "L'abbé Jean-Baptiste Gaultier (1685-1755) fut un adversaire acharné des Philosophes. Il est, entre autres, l'auteur d'un essai sur Pope, Le Poème de Pope, intitulé "Essay sur l'homme convaincu d'impiété" (1746) et d'un ouvrage célèbre contre Montesquieu, Les Lettres persanes convaincues d'impiété (1751). »

70. M.-F. Loquet, Cruzamante, op. cit., p. 131. Nous soulignons.

71. «Efforcez-vous d'entrer par la porte étroite», Luc, $13: 24$.

72. Fragments spirituels, III, OC, tome I, p. 779.

73. C'est lui que Fénelon cite le plus dans l'Explication des Maximes des saints.

74. La Tradition secrète des mystiques ou Le Gnostique de saint Clément d'Alexandrie [1694], éd. D. Tronc et M. Tronc, Paris, Arfuyen, 2006.

75. M.-F. Loquet, Cruzamante, op. cit., p. 135.

76. Ibid., p. 405.

77. Ibid., p. 410. Nous soulignons.

78. Si la Prudence est une vertu cardinale, dans Cruzamante, la Prudence humaine désigne un manque d'engagement de l'âme dévote pour son créateur.

79. Ibid., p. 298.

80. Il est fait ici référence à l'Explication des Maximes des saints de Fénelon et à sa condamnation papale en 1699.

81. M. Leprince de Beaumont, Les Américaines, op. cit., tome V, p. 64-65.

82. Matthieu, $27: 46$; Marc, $15: 34$.

83. M.-F. Loquet, Cruzamante, op. cit., p. 211.

84. Ibid., p. 422.

85. Ibid., p. 123.

86. Ibid., p. 355.

87. Ibid., p. 454.

88. F. Preyat, « LOQUET, MARIE-FRANÇOISE (1750-18..) », op. cit., p. 987.

89. J. Deprun, La Philosophie de l'inquiétude en France au XVIII siècle, Paris, Vrin, 1979, p. 165-166, cité par F. Preyat, op. cit., p. 991.

90. Voir cette remarque de Fabrice Preyat, extraite de la présentation du Séminaire du GEMCA (Louvain-la-Neuve, Collège Érasme, local ERAS 74, 19 octobre 2017) : "Souffrir est la marque certaine du véritable amour", Littérature emblématique et pédagogie mortifère chez MarieFrançoise Loquet. »

91. M.-F. Loquet, Cruzamante, op. cit., p. 174.

92. Dans la rhétorique antique, l'insinuatio repose sur le détour, la dissimulation (dissimulatio) et la longueur (laboriosa praelocutio chez Sulpitius Victor, dans Rhetores latini minores, éd. Halm, Leipzig, Teubner, 1863, p. 322, l. 15 ; et longiora principia chez Aurelius Augustin, ibid., p. 150, 1. 18). 
Pour la dissimulatio, voir Cicéron, De L'Invention, I, 24, trad. G. Achard, Paris, Les Belles Lettres, 1994, p. 79 ; Rhétorique à Herennius, I, 9-11, trad. G. Achard, Paris, Les Belles Lettres, 1989, p. 8-11.

93. Voir les chapitres consacrés à l'insinuation dans A. Mezzadri-Guedj, Pur Amour, pur style? Fénelon : du paradoxe à la répétition, Paris, Classiques Garnier, à paraître.

94. M.-F. Loquet, op. cit., p. 77.

95. Ibid., p. 295.

96. Ibid., p. 434.

97. Ibid., p. 57.

98. Ibid., p. 50. 